\title{
Clinical Characteristics of Critically Ill Patients Infected with COVID-19 in Rasoul Akram Hospital in Iran: A Single Center Study
}

\author{
Poupak Rahimzadeh (iD ${ }^{1}$, Saied Amniati ${ }^{2}$, Reza Farahmandrad ${ }^{2}$, Seyed Hamid Reza Faiz ${ }^{2,{ }^{*}}$, Setareh \\ Hedayati Emami ${ }^{3}$ and Azadeh Habibi ${ }^{3}$ \\ ${ }^{1}$ Pain Research Center, Department of Anesthesiology and Pain Medicine, Iran University of Medical Sciences, Tehran, Iran \\ ${ }^{2}$ Rasoul Akram Hospital Clinical Research Development Center (RCRDC), Iran University of Medical Sciences, Tehran, Iran \\ ${ }^{3}$ Rasoul Akram Hospital, Iran University of Medical Sciences, Tehran, Iran \\ Corresponding author: Rasoul Akram Hospital Clinical Research Development Center(RCRDC), Iran University of Medical Sciences, Tehran, Iran. Email: \\ shr.faiz.anesthesiology@gmail.com
}

Received 2020 July 06; Revised 2020 August 03; Accepted 2020 August 25.

\begin{abstract}
Background: Knowledge about clinical features of critically ill patients with COVID-19 still lacks adequate information up to now. Objectives: We aimed to describe and compare the epidemiological and clinical characteristics of critically ill patients with COVID19 in Rasoul Akram Hospital.

Methods: In this case series, 70 critically ill patients with COVID-19 admitted in ICU wards of Rasoul Akram Hospital, Tehran, Iran, from 29 February to 25 April 2020 were enrolled. Demographic and clinical characteristics, laboratory data, and outcomes of the patients were all collected and compared between deceased and recovered patients.

Results: Fifty-six cases had died of COVID-19, and 14 patients had fully recovered and discharged. The median age of the patients was 68 years old, ranging from 22 to 91 years, $66 \%$ were men, $80 \%$ had one or more comorbidities, and hypertension was the most common comorbidities ( $45 \%$ of deceased cases). The most common signs and symptoms at the onset of illness were $\mathrm{SPO}_{2}$ depression (92\%) and dyspnea (90\%). Dyspnea was significantly more common in deceased patients $(95 \%)$ than recovered patients $(70 \%)(\mathrm{P}=$ 0.048). Most patients had lymphopenia (80\%). The number of patients who needed mechanical ventilation in the deceased patients was 53 (95\%), which was significantly more than the recovered patients $(10[70 \%])(\mathrm{P}=0.048)$.

Conclusions: The mortality rate of critically ill patients with COVID-19 is very high, and the patients with dyspnea and required mechanical ventilation are at higher risk for death.
\end{abstract}

Keywords: COVID-19, ICU, Critically Ill, Pandemic, Mortality

\section{Background}

In December 2019, a cluster of pneumonia cases with an unknown cause and similar history of visiting a local seafood market were identified in Wuhan, Hubei Province, China, and their clinical manifestations were similar to viral pneumonia $(1,2)$. Deep sequencing analysis and realtime reverse transcription PCR (RT-PCR) from lower respiratory tract samples detected a new coronavirus, which was distinct from severe acute respiratory syndrome coronavirus "SARS-CoV" (identity of about 79\%) and Middle East respiratory syndrome coronavirus "MERS-CoV" (identity of about 50\%) (3). The World Health Organization (WHO) officially named it Coronavirus disease-19 (COVID-19) on 11 February 2020 (4). The number of confirmed patients with COVID-19 rapidly grew up in China and thereafter spread throughout the world, and WHO declared COVID-19 as a pandemic on 11 March 2020. It probably seems that COVID19 is the greatest threat to human societies in the 21st century (5).

Common signs and symptoms of COVID-19 include fever, sore throat, cough, malaise, headache, nausea, vomiting, diarrhea, and breathlessness (5). The clinical manifestations of COVID-19 are widely heterogeneous, ranging from asymptomatic infection (with no or mild pneumonia) to severe pneumonia with respiratory failure and multi-organ dysfunction or death $(6,7)$. Unlike SARS-CoV, patients infected with COVID-19 can transmit the disease and contribute to the spread of infection during the prodromal period with mild symptoms; therefore, the virus spread worldwide shortly after the first detection (8). Until 6 July 2020, the last update shows that over 4.5 million cases with laboratory-confirmed with COVID-19 have been detected in the world, and Iran is among the top 10 coun- 
tries in terms of infected patients and mortality due to COVID-19 (9).

Since the disease has a highly contagious nature and potential mortality (10), understanding all clinical implications of the virus will help to improve disease management, thereby controlling the pandemic. Most previous studies have just reported the general epidemiological findings, clinical characteristics, and clinical outcomes of patients with COVID-19 (3, 5-7), and there is a paucity of specific information about the characteristics of critically ill patients with COVID-19.

\section{Objectives}

To the best of our knowledge, there is no study focusing on critically ill patients infected with COVID-19 in Iran. Therefore, this study aim at describing and comparing the epidemiological and clinical characteristics of critically ill patients with COVID-19 who died or survived.

\section{Methods}

\subsection{Study Population}

The present study was designed as a case series, one which was performed on critically ill patients of COVID-19 admitted in ICU wards of Rasoul Akram Hospital, Tehran, Iran, from 29 February to 25 April 2020. All patients with COVID-19 enrolled in this study were diagnosed based on the WHO interim guidance (11), and nasal and pharyngeal swab specimens were collected for extracting SARS-CoV-2 RNA from the patients by Real-time polymerase chain reaction (RT-PCR). According to clinical practice guidelines from the Infectious Diseases Society of America and the American Thoracic Society for diagnosis and treatment of adults with community-acquired pneumonia (last updated 2019)(12), critically ill patients of COVID-19 were classified for ICU care. The study protocol was approved by the institutional review board (IRB) of Iran University of Medical Sciences, and the Ethics Committee approved the study before its initiation (ethics code: IR.IUMS.REC.1399.037).

\subsection{Data Collection}

Demographic and clinical characteristics and laboratory data of the patients, including age, gender, smoking history, comorbidities, clinical signs and symptoms, history of annual influenza vaccination, mechanical ventilation, hemodynamic status, peripheral capillary oxygen saturation $\left(\mathrm{SPO}_{2}\right)$, complete blood count (CBC), lactate dehydrogenase (LDH), D-dimer, and Arterial blood gas (ABG) were obtained from their medical records upon admission using a standardized data collection form. Moreover, patients' outcomes (death or survival) were extracted from medical records.

\subsection{Statistical Analysis}

The statistical software package SPSS for Windows, version 20.0 (SPSS, Chicago, IL, USA) was used for data analysis. Descriptive analyses of the variables were expressed as mean ( \pm standard deviation $=S D$ ), median (with an interquartile range $=\mathrm{IQR}=\mathrm{Q1}-\mathrm{Q} 3$ ), or number $(\%)$. The clinical characteristics of both groups (death or Survival) were compared using $\chi^{2}$ tests or Fisher's exact test for proportions and unpaired $t$-tests for means. Pearson correlation coefficient was calculated to evaluate the association between characteristics in each group. A P value of $<0.05$ was considered statistically significant.

\section{Results}

From 29 February to 25 April 2020, the number of 70 critically ill patients with laboratory-confirmed COVID-19 was admitted to the ICU of Rasoul Akram Hospital. Overall, 56 cases had died of COVID-19, and 14 patients had fully recovered and been discharged. The median age of the patients was 68 years old $(\mathrm{IQR}=57 \cdot 0-77 \cdot 0)$, ranging from 22 to 91 years. Gender distribution (male/female) of deceased and recovered patients was 36/20 and 10/4, respectively ( $\mathrm{P}$ $=1.00$ ). Fifty-six patients ( $80 \%$ ) had one or more comorbidities, and hypertension was the most common comorbidities between them, $50 \%$ of infected patients and $45 \%$ of deceased cases had hypertension. History of cigarette smoking was more predominant in deceased patients $(11 ; 20 \%)$ than in recovered patients (0;0\%); however, its difference was not statistically significant $(\mathrm{P}=0.184)$. The baseline characteristics of the patients are represented in Table 1. There were no statistically significant differences between deceased and recovered patients regarding baselines.

Overall, the most common signs and symptoms at the onset of illness were SPO2 saturation drop (64 [92\%]), dyspnea (63 [90\%]), followed by cough (56 [80\%]), and fever (56 [80\%]); less common signs and symptoms were myalgia (29 [42\%]), sore throat (11 [16\%]), diarrhea (8 [12\%]), and nausea and vomiting (6 [8\%]). Dyspnea was significantly more common in non-survivors 53 (95\%) compared with recovered patients $10(70 \%)(\mathrm{P}=0.048)$. We found cough and fever in $77.5 \%$ of non-survivors compared with $90 \%$ in recovered patients $(\mathrm{P}=0.66)$. However, there were no statistically significant differences between survivor and nonsurvivor regarding other signs and symptoms at the onset of illness (Table 2). 


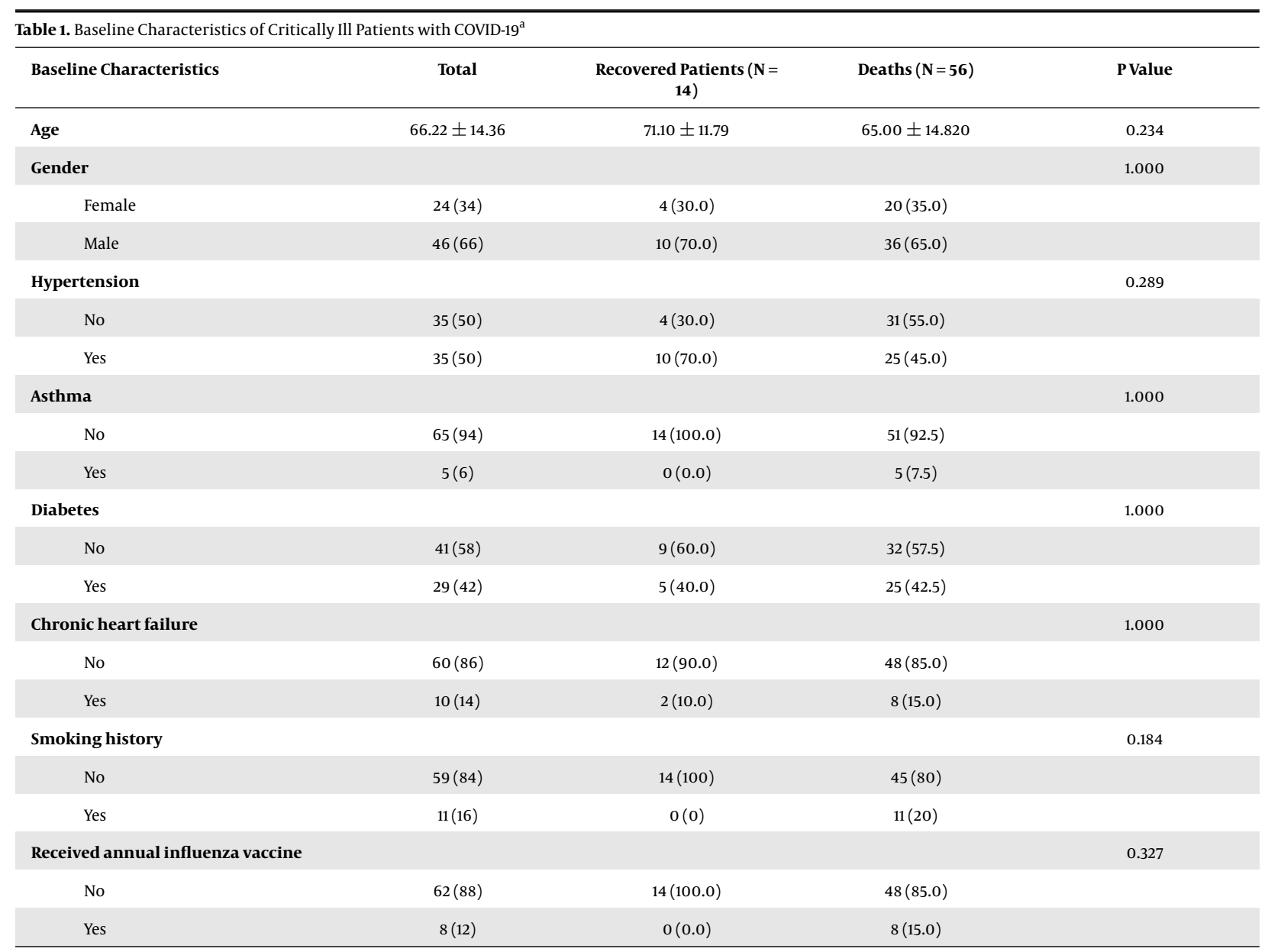

${ }^{\mathrm{a}}$ Values are expressed as No. (\%) or mean $\pm \mathrm{SD}$.

The median duration of fever was 12 and 13 days in survivors and non-survivors, respectively. The median duration of cough persisted for 16 days and 13 days in survivors and non-survivors, respectively. Seven (50\%) survivors still had a cough on discharge. Moreover, $95 \%$ of non-survivors and $70 \%$ of survivors were intubated $(\mathrm{P}=0.048)$. Among the deceased patients, the median time from hospital admission to mechanical ventilation was 3.8 days, which was similar to recovered patients (4.2 days). The median time of ICU stay was 10 days for non-survivors and 6.5 days for survivors. The median time of invasive mechanical ventilation need in ICU was 6.5 and 4 days for deceased and survived patients, respectively ( $\mathrm{P} \geq 0.05)$.

Among non-survivors, the median time from illness onset was 9,10, and 11 days to sepsis, ARDS, and secondary infection, respectively. Among survivors, two cases of secondary infection and acute kidney injury were observed, which occurred 7 and 10 days after illness onset, respectively.
Clinical and laboratory findings of the patients were recorded on the day of hospital admission for all patients (Table 3). The blood counts of patients on hospital admission showed that only 17/70 patients had anemia. Most of them had lymphopenia (lymphocyte count $<1.0 \times 10^{9} / \mathrm{L}$; 56 (80\%) patients); however, it was not significant between the two groups. The mean LDH levels on admission were higher in the deceased $(1.073 .68 \pm 293.50)$ than the recovered patients ( $970.60 \pm 205.98$ ), but the difference was not significant $(\mathrm{P}=0.519)$. All of the patients had D-dimer $\geq$ $0.5(\mathrm{P}=0.00)$. Hemodynamic instability (need to receive vasopressor on admission) was recorded in 3 (5\%) of deceased patients, while all of the recovered patients had stable hemodynamic on the time of hospital admission. In addition, the mean of peripheral oxygen saturation $\left(\mathrm{SpO}_{2}\right)$ in deceased patients was $62.80 \pm 12.68$, which was slightly lower than that of recovered patients $(70.14 \pm 8.37)$.

The Pearson correlations of $\mathrm{SPO}_{2}$ with $\mathrm{PCO}_{2}, \mathrm{PaO}_{2}, \mathrm{PH}$, duration of mechanical ventilation, and respiratory rate 


\begin{tabular}{|c|c|c|c|c|}
\hline $\begin{array}{l}\text { Signs and Symptoms Upon Hospital } \\
\text { Admission }\end{array}$ & Total & $\begin{array}{l}\text { Recovered Patients }(\mathrm{N}= \\
\text { 14) }\end{array}$ & Deaths $(N=56)$ & PValue \\
\hline Fever & & & & 0.663 \\
\hline No & $14(20)$ & $2(10.0)$ & $12(22.5)$ & \\
\hline Yes & $56(80)$ & $12(90.0)$ & $44(77.5)$ & \\
\hline Dyspnea & & & & 0.048 \\
\hline No & $7(10)$ & $4(30.0)$ & $3(5.0)$ & \\
\hline Yes & $63(90)$ & $10(70.0)$ & $53(95.0)$ & \\
\hline $\mathbf{S P O}_{2}<\mathbf{9 3} \%$ & & & & 1.000 \\
\hline No & $6(8)$ & $2(10.0)$ & $4(7.5)$ & \\
\hline Yes & $64(92)$ & $12(90.0)$ & $52(92.5)$ & \\
\hline Myalgia or fatigue & & & & 0.488 \\
\hline No & $41(58)$ & $10(70.0)$ & $31(55.0)$ & \\
\hline Yes & $29(42)$ & $4(30.0)$ & $25(45.0)$ & \\
\hline Nausea and vomiting & & & & 0.571 \\
\hline No & $64(92)$ & $14(100.0)$ & $50(90.0)$ & \\
\hline Yes & $6(8)$ & $0(0.0)$ & $6(10.0)$ & \\
\hline Cough & & & & 0.663 \\
\hline No & $14(20)$ & $2(10.0)$ & $12(22.5)$ & \\
\hline Yes & $56(80)$ & $12(90.0)$ & $44(77.5)$ & \\
\hline Diarrhea & & & & 0.327 \\
\hline No & $62(88)$ & $14(100.0)$ & $48(85.0)$ & \\
\hline Yes & $8(12)$ & $0(0.0)$ & $8(15.0)$ & \\
\hline Sore throat & & & & 1.000 \\
\hline No & $59(84)$ & $11(80.0)$ & $48(82.5)$ & \\
\hline Yes & $11(16)$ & $3(20.0)$ & $8(17.5)$ & \\
\hline
\end{tabular}

${ }^{\mathrm{a}}$ Values are expressed as No. (\%).

was tested for deceased and recovered patients and the results of which are shown in Table 4 . In the recovered group, no significant relationship was found between these variables $(\mathrm{P}>0.05)$. In the deceased group, there is an inverse relationship only between $\mathrm{SPO}_{2}$ with respiratory rate $(\mathrm{r}=$ $0.326, \mathrm{P}=0.040$ ), whereas the relationship between other variables with $\mathrm{SPO}_{2}$ was not significant $(\mathrm{P}>0.05)$.

\section{Discussion}

The mainstay of treatment for COVID-19 is supportive care to control symptoms and protects important organs due to lack of vaccine and specific antiviral medication for effective treatment at this time. It seems that close followup and supportive care are sufficient to manage the disease, but aggressive treatments and intensive care units for critically ill patients are needed (13). Up to now, limited studies have specifically focused on critically ill patients with COVID-19, and all three previously published studies of critically ill patients had a small sample size (14-16). In the present study, we report clinical features and outcomes of 70 critically ill patients with confirmed COVID-19 who admitted to ICU.

In agreement with the previous reports, $66 \%$ of critical cases infected by COVID-19 were men. In the Chinese cohort study (14), nearly 70\% of critically ill patients were men, and it was 63\% in the American study (16). Based on pieces of evidence, male patients are more susceptible to COVID19 and seem to be the most prone to severe conditions. Our results showed these findings as well. However, there is no significant difference between survivors and nonsurvivors regarding gender distribution. As mentioned in previous studies, a large proportion of patients (80\%) had one or more comorbidities, and hypertension was the 


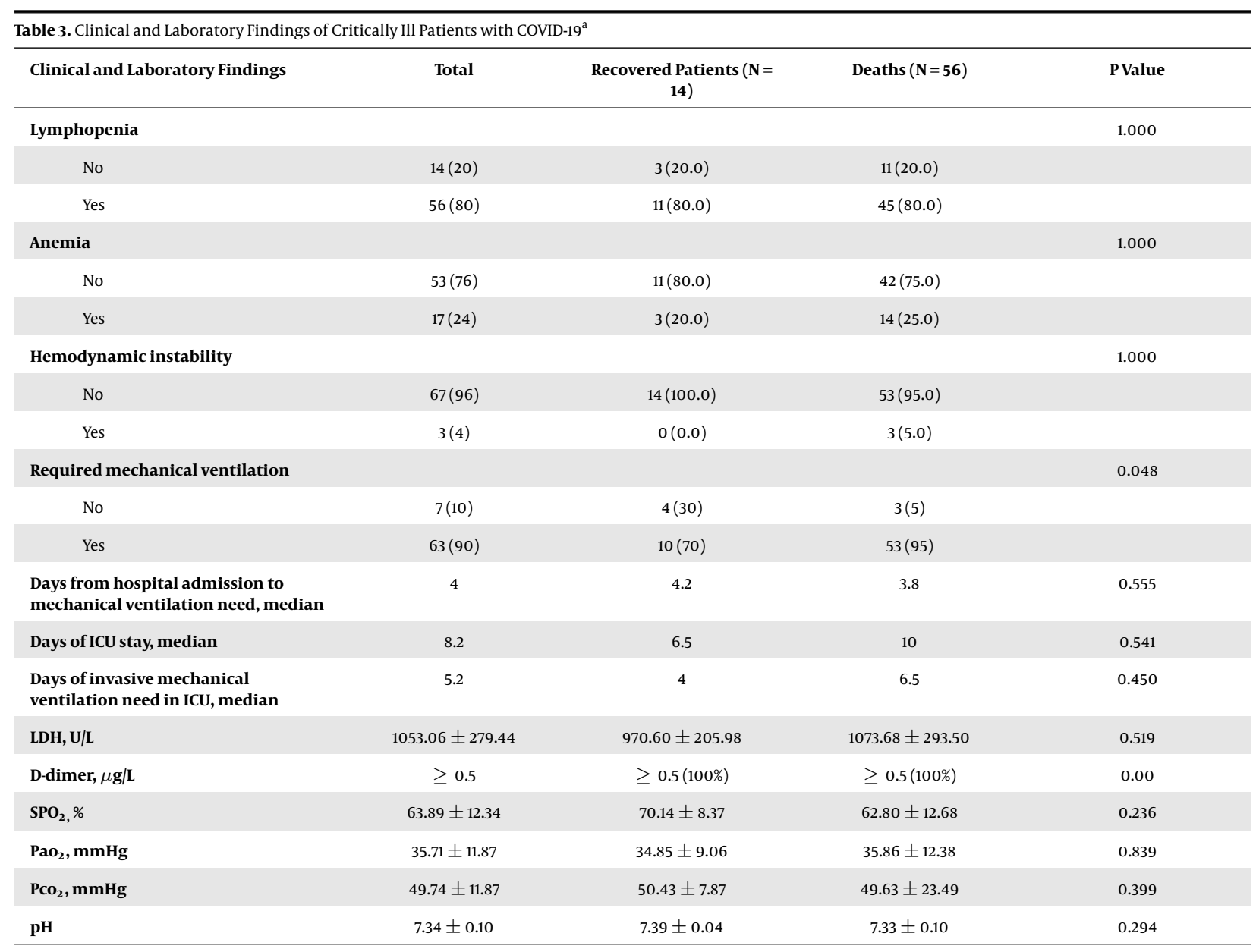

${ }^{\mathrm{a}}$ Values are expressed as No. (\%) or mean $\pm \mathrm{SD}$.

most common comorbidity between the patients (17). We found hypertension as the most prevalent comorbidity as well. Moreover, we noted that the rate of comorbidities did not differ between survivors and non-survivors.

The mortality rate in our study was $80 \%$ that was higher than the previous studies. In an American study, the mortality rate was $50 \%(16)$, and it was $61.5 \%$ in a chines study regarding critically ill patients (15). Both mentioned studies followed the patients for 14 and 28 days, and some patients remained in ICU or intubated at the time of data gathering.

Clinical characteristics of COVID-19 are nearly similar to previous coronavirus infections. In our study, $\mathrm{SPO}_{2}$ decline, dyspnea, cough, and fever were the most common symptoms in the time of admission in critically ill patients with COVID-19. Consistent with our results, other studies reported that these symptoms are the most common among critical patients with COVID-19 pneumonia (17). Moreover, similar to previous studies, intestinal signs and symptoms were less common (diarrhea $12 \%$, nausea, and vomiting $8 \%$ ) among the patients of our study, whereas intestinal signs and symptoms have been presented in about $20 \%-25 \%$ of patients with MERS-CoV or SARS-CoV infection $(18,19)$. Comparing non-survivors with survivors in the current study, dyspnea was significantly more common in deceased patients; however, other prevalent symptoms were similar in both deceased and recovered patients.

According to laboratory tests, lymphopenia was seen in $80 \%$ of critically ill patients, and it was concordant with the results from the previous studies. However, there is no difference between survivors and deaths. This is consistent with the report of Yang et al. (14), who showed that more than $80 \%$ of critically ill patients had lymphopenia, and it was $75 \%$ in case series done in the USA (16). Lymphopenia is a common characteristic of critically ill patients with SARS-CoV infection because SARS-CoV viral particles play a destructive role in damaging the cytoplasmic component of the lymphocytes (20). In addition, owing 


\begin{tabular}{|c|c|c|c|c|c|c|}
\hline \multirow{2}{*}{ Characteristics } & \multicolumn{6}{|c|}{ Correlations } \\
\hline & $\mathrm{PcO}_{2}$ & $\mathrm{PaO}_{2}$ & LDH & $\begin{array}{c}\text { Duration of } \\
\text { Mechanical } \\
\text { Ventilation, d }\end{array}$ & pH & Respiratory Rate \\
\hline \multicolumn{7}{|l|}{ Recovered patients } \\
\hline \multicolumn{7}{|l|}{$\mathrm{SPO}_{2}$} \\
\hline $\begin{array}{l}\text { Pearson } \\
\text { correlation }\end{array}$ & 0.613 & 0.446 & 0.321 & 0.240 & 0.280 & 0.356 \\
\hline Pvalue & 0.143 & 0.316 & 0.483 & 0.605 & 0.543 & 0.434 \\
\hline Numbers & 14 & 14 & 14 & 14 & 14 & 14 \\
\hline \multicolumn{7}{|l|}{ Deaths } \\
\hline \multicolumn{7}{|l|}{$\mathrm{SPO}_{2}$} \\
\hline $\begin{array}{l}\text { Pearson } \\
\text { correlation }\end{array}$ & -0.005 & $0.292^{\mathrm{a}}$ & -0.094 & -0.032 & $0.280^{a}$ & $-0.326^{b}$ \\
\hline Pvalue & 0.976 & 0.068 & 0.562 & 0.844 & 0.080 & 0.040 \\
\hline Numbers & 56 & 56 & 56 & 56 & 56 & 56 \\
\hline
\end{tabular}

to the induced apoptosis of lymphocytes by the MERS-CoV virus, lymphopenia is a prominent feature in critically ill patients with MERS infection (21, 22). Therefore, it creates a hypothesis that lymphopenia in critically ill patients with COVID-19 could be caused by virus-induced necrosis or apoptosis in lymphocytes. In a previous study, which reported the characteristics of non-critical patients infected with COVID-19, only $35 \%$ of patients had mild lymphopenia (23), suggesting that the severity of lymphopenia may the probable reason for the severity of COVID-19.

Mechanical ventilation plays an essential role as a supportive treatment for critically ill patients. In our study, most of the patients (90\%) needed mechanical ventilation. This finding was along with the results of other studies. In the study conducted by Arentz et al. (15), 71\% of critically ill patients needed mechanical ventilation, and it was $75 \%$ in another study by Bhatraju et al. (16). Additionally, the rate of required mechanical ventilation in non-survivors was significantly more than the recovered. This is similar to Yang et al. (14), who showed that 57\% of non-survivors required mechanical ventilation, but it was 35\% among survivors.

There were a number of limiting factors, diminishing the impact of results of which the patient population in a single referral center is the most important one. Thus our results may not be representative of patients in Iran with this condition due to the retrospective study design, not all laboratory tests were done in all patients, including procalcitonin, IL-6, and serum ferritin. Also, since we are involved in the management of critically ill patients with
COVID-19 in one hospital, our sample size was small. In addition, some specific information from the ICU, including mechanical ventilation settings, radiological findings, and supportive treatments and interventions, are missed. Instead, we hope that our efforts will encourage a larger and multicenter investigation for a better understanding of clinical features, risk factors, and outcomes of COVID-19.

\subsection{Conclusions}

Our findings provide some important clinical characteristics of critically ill patients with COVID-19 admitted to ICU and needed ICU care and ventilatory support. The mortality rate of critically ill patients with COVID-19 is very high, and the patients with dyspnea and required mechanical ventilation are at higher risk for poor outcomes and death after ICU admission. We found that male hypertensive gender with dyspnea at admission were risk factors for ICU-admission of adult patients with COVID-19. However, more extensive large multicenter studies should be performed to define all risk factors and specify more effective care for these challenging patients.

\section{Acknowledgments}

The authors wish to thank Rasoul Akram Hospital Clinical Research Development Center (RCRDC), Iran University of Medical Science, for technically supported implementation of the project. 


\section{Footnotes}

Authors' Contribution: All authors agree to be accountable for all aspects of the work in ensuring that questions related to the accuracy or integrity of any part of the work are appropriately investigated and resolved. Study concept and design: PR. Acquisition of data: SHRF, SA, and AH. Drafting the manuscript: PR and RF. Critical revision of the manuscript for important intellectual content: SHE. Study supervision: PR and SHRF.

Conflict of Interests: The authors have no conflict of interest to declare.

Ethical Approval: The study protocol was approved by the institutional review board (IRB) of Iran University of Medical Sciences, and the Ethics Committee approved the study before its initiation (ethics code: IR.IUMS.REC.1399.037)

Funding/Support: It was not declared by the authors. Informed Consent: Informed consent was obtained from all individuals.

\section{References}

1. Zhu N, Zhang D, Wang W, Li X, Yang B, Song J, et al. A novel coronavirus from patients with pneumonia in China, 2019. N Engl J Med. 2020;382(8):727-33. doi:10.1056/NEJMoa2001017. [PubMed: 31978945]. [PubMed Central: PMC7092803].

2. Gralinski LE, Menachery VD. Return of the coronavirus: 2019nCoV. Viruses. 2020;12(2). doi: 10.3390/v12020135. [PubMed: 31991541]. [PubMed Central: PMC7077245].

3. Lu R, Zhao X, Li J, Niu P, Yang B, Wu H, et al. Genomic characterisation and epidemiology of 2019 novel coronavirus: Implications for virus origins and receptor binding. The Lancet. 2020;395(10224):565-74. doi: 10.1016/s0140-6736(20)30251-8.

4. World Health Organization. Naming the coronavirus disease (COVID-19) and the virus that causes it. WHO; 2020.

5. Wu Z, McGoogan JM. Characteristics of and important lessons from the coronavirus disease 2019 (COVID-19) outbreak in China: Summary of a report of 72314 cases from the Chinese Center for Disease Control and Prevention. JAMA. 2020;323(13):1239-42. doi: 10.1001/jama.2020.2648. [PubMed: 32091533].

6. Zheng YY, Ma YT, Zhang JY, Xie X. COVID-19 and the cardiovascular system. Nat Rev Cardiol. 2020;17(5):259-60. doi:10.1038/s41569-020-03605. [PubMed: 32139904].

7. Chan JFW, Yuan S, Kok KH, To KKW, Chu H, Yang J, et al. A familial cluster of pneumonia associated with the 2019 novel coronavirus indicating person-to-person transmission: A study of a family cluster. Lancet. 2020;395(10223):514-23. doi:10.1016/s0140-6736(20)30154-9.

8. Alimohamadi Y, Taghdir M, Sepandi M. Estimate of the basic reproduction number for COVID-19: A systematic review and meta-analysis. J Prev Med Public Health. 2020;53(3):151-7. doi: 10.3961/jpmph.20.076. [PubMed: 32498136].

9. Worldometers. Iran demographics. 2020. Available from: https://www. worldometers.info/world-population/iran-population/.

10. Yeo C, Kaushal S, Yeo D. Enteric involvement of coronaviruses: Is faecal-oral transmission of SARS-CoV-2 possible? Lancet Gastroen- terol Hepatol. 2020;5(4):335-7. doi: 10.1016/s2468-1253(20)30048-0. [PubMed: 32087098]. [PubMed Central: PMC7130008].

11. WHO. Clinical management of severe acute respiratory infection when Novel coronavirus ( $\mathrm{nCoV}$ ) infection is suspected: Interim guidance. 2020, [cited 2020 Mar 10]. Available from: https://www.who.int/internalpublications-detail/clinicalmanagement-of-severe-acute-respiratory-infection-when-novelcoronavirus-(ncov)-infection-is-suspected.

12. Metlay JP, Waterer GW, Long AC, Anzueto A, Brozek J, Crothers K, et al. Diagnosis and Treatment of Adults with Community-acquired Pneumonia. An Official Clinical Practice Guideline of the American Thoracic Society and Infectious Diseases Society of America. Am J Respir Crit Care Med. 2019;200(7):e45-67. doi: 10.1164/rccm.201908-1581ST. [PubMed: 31573350]. [PubMed Central: PMC6812437].

13. Murthy S, Gomersall CD, Fowler RA. Care for critically Ill patients with COVID-19. Jama. 2020;323(15). doi: 10.1001/jama.2020.3633. [PubMed: 32159735].

14. Yang X, Yu Y, Xu J, Shu H, Xia J, Liu H, et al. Clinical course and outcomes of critically ill patients with SARS-CoV-2 pneumonia in Wuhan, China: A single-centered, retrospective, observational study. Lancet Respir Med. 2020;8(5):475-81. doi: 10.1016/s2213-2600(20)30079-5.

15. Arentz M, Yim E, Klaff L, Lokhandwala S, Riedo FX, Chong M, et al. Characteristics and outcomes of 21 critically Ill patients with COVID-19 in Washington State. Jama. 2020;323(16):1612-4. doi: 10.1001/jama.2020.4326. [PubMed: 32191259]. [PubMed Central: PMC7082763].

16. Bhatraju PK, Ghassemieh BJ, Nichols M, Kim R, Jerome KR, Nalla AK, et al. Covid-19 in critically Ill patients in the Seattle Region - case series. N Engl J Med. 2020;382(21):2012-22. doi: 10.1056/NEJMoa2004500. [PubMed: 32227758]. [PubMed Central: PMC7143164].

17. Fu L, Wang B, Yuan T, Chen X, Ao Y, Fitzpatrick T, et al. Clinical characteristics of coronavirus disease 2019 (COVID-19) in China: A systematic review and meta-analysis. J Infect. 2020;80(6):656-65. doi: 10.1016/j.jinf.2020.03.041. [PubMed: 32283155]. [PubMed Central: PMC7151416].

18. Assiri A,Al-TawfiqJA,Al-Rabeeah AA,Al-Rabiah FA, Al-Hajjar S, Al-Barrak A, et al. Epidemiological, demographic, and clinical characteristics of 47 cases of Middle East respiratory syndrome coronavirus disease from Saudi Arabia: A descriptive study. Lancet Infect Dis. 2013;13(9):75261. doi: 10.1016/s1473-3099(13)70204-4. [PubMed: 23891402]. [PubMed Central: PMC7185445].

19. Lee N, Hui D, Wu A, Chan P, Cameron P, Joynt GM, et al. A major outbreak of severe acute respiratory syndrome in Hong Kong. $N$ Engl J Med. 2003;348(20):1986-94. doi: 10.1056/NEJMoa030685. [PubMed: 12682352].

20. Gu J, Gong E, Zhang B, Zheng J, Gao Z, Zhong Y, et al. Multiple organ infection and the pathogenesis of SARS. J Exp Med. 2005;202(3):41524. doi: 10.1084/jem.20050828. [PubMed: 16043521]. [PubMed Central: PMC2213088].

21. Chu H, Zhou J, Wong BH, Li C, Chan JF, Cheng ZS, et al. Middle east respiratory syndrome coronavirus efficiently infects human primary t lymphocytes and activates the extrinsic and intrinsic apoptosis pathways. J Infect Dis. 2016;213(6):904-14. doi: 10.1093/infdis/jiv380. [PubMed: 26203058]. [PubMed Central: PMC7107330].

22. Liu WJ, Zhao M, Liu K, Xu K, Wong G, Tan W, et al. T-cell immunity of SARS-CoV: Implications for vaccine development against MERSCoV. Antiviral Res. 2017;137:82-92. doi: 10.1016/j.antiviral.2016.11.006. [PubMed: 27840203]. [PubMed Central: PMC7113894].

23. Chen N, Zhou M, Dong X, Qu J, Gong F, Han Y, et al. Epidemiological and clinical characteristics of 99 cases of 2019 novel coronavirus pneumonia in Wuhan, China: A descriptive study. Lancet. 2020;395(10223):507-13. doi: 10.1016/s0140-6736(20)30211-7. [PubMed: 32007143]. [PubMed Central: PMC7135076]. 